http://jmscr.igmpublication.org/home/ ISSN (e)-2347-176x ISSN (p) 2455-0450

crossref DOI: https://dx.doi.org/10.18535/jmscr/v8i2.33

\title{
A Rare Case of Spinal Osteosarcoma with Extraosseous Component: Review of Literature for Role and Outcome of Radiation Therapy
}

\author{
Authors \\ Sameer Kr Verma ${ }^{1}$, Ajeet K Gandhi' ${ }^{2}$ Madhup Rastogi ${ }^{3}$, Satyajeet Rath ${ }^{4}$, \\ Sambit S Nanda ${ }^{5}$, Siddharth Kumar ${ }^{6}$, Ayushi Patni ${ }^{7}$ \\ Department of radiation Oncology, Dr. Ram Manohar Lohia Institute of Medical Sciences, \\ Vibhuti Khand, Gomti Nagar, Lucknow-226010 \\ Corresponding Author \\ Ayushi Patni
}

\begin{abstract}
Osteosarcoma (OS) most commonly occurs in metaphysis of long bones in young people. Spinal OS is a relatively uncommon tumor and difficult to treat owing to its close proximity to spinal cord. A 36 years old male with complaint of lower back pain for 3 months was diagnosed as a case of spinal OS of D12 vertebra with an extraosseous soft tissue component on radiological imaging and histopathological examination. The patient underwent D12, partial D11 and L1 laminectomy with tumour removal. Post operative imaging showed gross residual disease. External beam radiotherapy (EBRT) of 2000 cGy in 5 fractions to D10-L1 was delivered initially. As patient responded well to EBRT, additional 2000 cGy in 10 fractions was given followed by chemotherapy with good clinical and radiological response and symptomatic relief. With multimodality approach and careful selection of these patients, good local control and palliation can be achieved with early institution of radiotherapy in these patients.

Keywords: Osteosarcoma, Spinal osteosarcoma, Extraosseous spinal osteosarcoma, Radiotherapy in spinal osteosarcoma.
\end{abstract}

\section{Introduction}

Osteosarcomas (OS) is the most common primary malignant tumour of the bone ${ }^{[1]}$, it is a high-grade tumour characterized by the uncontrolled proliferation of spindle cells of mesenchymal origin leading to the production of osteoid or immature bone from malignant cells ${ }^{[2],[3]}$. Despite being the most common type of bone tumour spine is the rarest site of osteogenic sarcomas and accounts for only $0.85 \%-3 \%$ of ${ }^{[3],[4]}$ all osteosarcomas, 3.6\%$14.5 \%{ }^{(3,4)}$ of primary spinal tumours and $3 \%-5 \%$ of all spinal malignancies ${ }^{[3]-[6]}$. The spinal OS have the predilection for sacral area $(30 \%)$ followed by the lumbar $(25 \%)$ and thoracic spine $(25 \%)^{[3]}$. Spinal OS shows a bimodal peak in the age of onset of the disease, first peak is more common in the adolescents and the younger age group with second peak in the elderly age group ${ }^{[3]}$.

The challenges faced by clinicians in the treatment of the spinal OS are, its lower incidence due to which there are no robust studies regarding its management, anatomic location and close proximity to the vital structures. As osteosarcoma is a relatively radioresistant tumour, the mainstay of the treatment are surgery and adjuvant chemotherapy. Since, adequate resection with acceptable margin is 
rarely achievable in cases of spinal OS, hence in such cases radiotherapy (RT) is reserved as definitive treatment, though it can also be used as an effective measure for palliation ${ }^{[7]}$. We intend to report a rare case of spinal OS who underwent postoperative RT in view of inadequate surgical resection.

\section{Case Report}

A 36 years old male with no medical co morbidities presented with complaint of lower back pain radiating to right knee for 3 months. He was evaluated with contrast enhanced magnetic resonance imaging (CEMRI) of dorso-lumbar spine which was suggestive of a lesion involving D12 vertebra and its posterior elements with an extra osseous soft tissue component in the epidural space (Figure 1). Biopsy was done from D12 lesion which was suggestive of vertebral osteogenic sarcoma, osteoblastic type. Then patient underwent D12, partial D11 and partial L1 laminectomy with tumour removal followed by screw fixation. Slide and block review of biopsy specimen showed pleomorphic tumour cells, round to polygonal shaped with pleomorphic hyperchromatic nuclei and moderate amount of eosinophilic cytoplasm along with osteoclastic giant cells and mitotic count of 1012/high power field with immunohistochemistry CD99 positive and S-100 negative and reported to be osteosarcoma, high grade, conventional type. Bone scan and computed tomography (CT) of the region confirmed similar findings. Patient received 1 cycle of cisplatin $60 \mathrm{mg} / \mathrm{m}^{2} /$ day and adriamycin $37.5 \mathrm{mg} / \mathrm{m}^{2} /$ day from D1 to D3. Patient had minimal response to chemotherapy with progression of paraparesis. Hence, external beam radiotherapy (EBRT) was given initially at doses of 2000 cGy in 5 fraction@4Gy per fraction and in view of good response was further given 2000 cGy in 10 fractions to field D10 to L1 with 3 dimensional-conformal radiotherapy technique (3D-CRT). Patient showed significant improvement in paraparesis after radiotherapy completion. This was followed by 1 more cycle of cisplatin and adriamycin based chemotherapy, followed by switch to ifosfamide and adriamycin based chemotherapy. He received 2 cycles of ifosfamide $2400 \mathrm{mg} / \mathrm{m}^{2} /$ day from D1 to D5 and adriamycin $37.5 \mathrm{mg} / \mathrm{m}^{2} /$ day from D1 to D3 at three weekly intervals. CECT whole abdomen done post chemotherapy is suggestive of area of rarefaction/destruction involving D12 vertebrae and an extra osseous soft tissue mass of size 71 x 50 x $49 \mathrm{~mm}$. MRI lumbo-sacral spine is suggestive of an ill-defined soft tissue area of intermediate signal intensity on $\mathrm{T} 1$ and $\mathrm{T} 2$ in the region of pedicles and laminae at D12 with subtle ill-defined enhancement adjacent to the left pedicle. Then patient received 4 cycles of ifosfamide $2400 \mathrm{mg} / \mathrm{m}^{2} /$ day from D1 to D5 and cisplatin $60 \mathrm{mg} / \mathrm{m}^{2} /$ day from D1 to D3 at three weekly interval. Post-treatment CECT whole abdomen is suggestive of mild D12 vertebral collapse with irregular lytic and sclerotic lesion involving posterior elements of D12 and no obvious associated extra osseous soft tissue mass lesion seen (Figure 2). On post-treatment follow-up patient had subjective response of $80 \%$ and was able to walk with support.

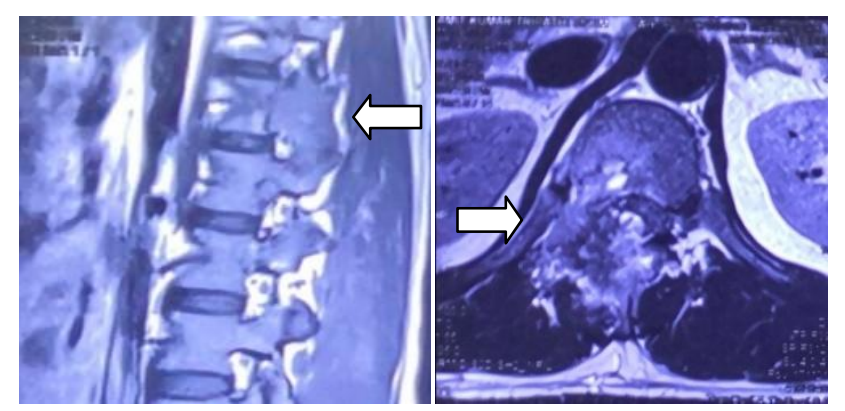

Fig 1. Pre-operative sagittal and axial views of MRI spine

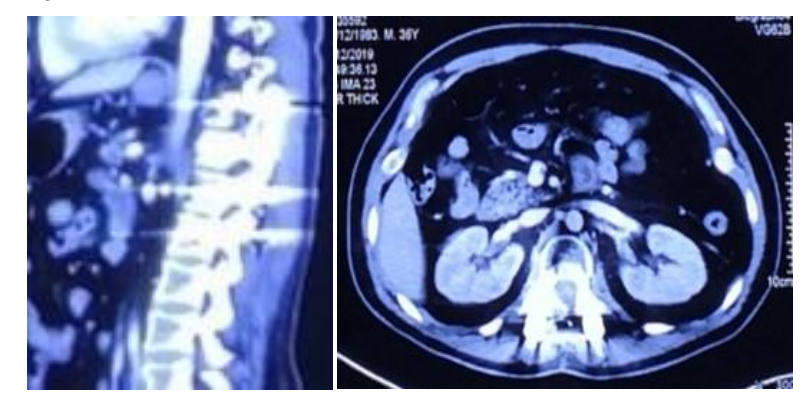

Fig 2. Post treatment completion sagittal and axial views of spine CT scan

\section{Discussion}

In spinal OS, $75 \%$ of the lesions in the vertebral body are malignant, whereas lesions originating in 
the posterior element, only one third are malignant. The most common presenting symptom of OS is pain at the local site, which is insidious in onset and intolerable and can occur during sleep as well. A palpable enlarging mass, restricted movement, pain on weight bearing and local warmth in the absence of any evidence of infection and injury, along with weight loss, malaise and fever, are suggestive of osteosarcoma $^{[2]}$. Pathological fractures may also occur in cases of spinal OS, which predominantly involves body of the vertebra in $90 \%$ cases $^{[8]}$. In due course of time as the tumour progresses it frequently involves the spinal canal and leads to cord compression which results in neurologic deficits and is associated with a poor prognosis ${ }^{[2],[3],[8]}$. Histologically, following subtypes of spinal osteosarcomas have been described, chondroblastic and osteoblastic type which is the most common type, small cell type, and fibroblastic type which is the least frequent type ${ }^{[2]}$. Classical forms of the osteosarcomas contains tumour cells of shapes and sizes varying from spindled to polyhedral with pleomorphic hyperchromatic nuclei in bone matrix ${ }^{[9]}$. World health organization (WHO) described histological grouping of osteosarcomas as conventional, telengeictatic, small cell, low grade central, secondary, paraosteal, periosteal and high grade surface types ${ }^{[10]}$. The conventional form of osteosarcomas are considered as the classic form which is a high grade primary malignant osteogenic tumour $^{[5]}$. The histologic grading has importance in staging of the disease and adjuvant treatment planning in the post-operative cases. Imaging of the spinal osteosarcomas also has importance in the treatment planning of the disease. Ilaslan $\mathrm{H}$ et $\mathrm{al}^{[11]}$ found that $79 \%$ of these tumours arise in the posterior elements of the vertebral body with partial body involvement, and $84 \%$ of the cases have spinal canal invasion in presence of high grade tumour. CT is considered superior to both plain radiographs and MR imaging in describing the mineralization pattern of the lytic lesions and cortical destruction of the involved spine ${ }^{[5]}$.

Due to infrequent occurrence of the tumour there are very few reports on treatment of spinal osteosarcoma. In cases of extremity osteosarcoma studies have reported that wide resection and neoadjuvant chemotherapy are associated with a significant improvement in the patient outcomes ${ }^{[8]}$, ${ }^{[12]}$. However, in case of the spinal osteosarcoma same benefit have not been reported, despite significant advances in diagnostic and treatment modalities $^{[1]}$. A number of advanced surgical techniques are available which enable the en bloc resection of the tumour. However, anatomical location in spine limits the ability of en bloc resection to achieve wide surgical margins, because of which local control with surgery may not be adequate. So, in these cases, non-surgical treatment such as chemotherapy and RT plays an important role. Some studies suggested that surgical techniques if applied in combination with chemotherapy and radiation have promising long term survival rates ${ }^{[3],[4],[8],[12]-[14]}$. Chemotherapy in neoadjuvant setting 6-12 weeks prior to surgery have improved response and survival rates as compared to surgery alone and post-operative chemotherapy alone ${ }^{[15]}$. Doxorubicin and cisplatin is considered as the basis of the treatment, although high-dose methotrexate and/or ifosfamide if combined, have additional benefit over two drug regimen ${ }^{[15]}$. Role of radiotherapy is limited in OS, but in selected cases and for palliation RT can be useful in achieving good local control. Depending upon the resectability of tumour and the response to the chemotherapy, radiotherapy may be delivered as neoadjuvant, adjuvant or primary local therapy ${ }^{[16]}$. Neoadjuvant radiotherapy can be considered for downsizing the tumour to facilitate the surgical resection, while adjuvant radiotherapy is given postoperatively in patients with positive or inadequate resection margins ${ }^{[17]}$. In medically inoperable disease, radiation therapy as the primary local therapy without surgery is considered useful in achieving good local control of the disease ${ }^{[3],[16]}$, but in these cases there are chances of significant tissue toxicity, as the radiation dose required is much higher to achieve the best local control ${ }^{[5]}$ and also radiation therapy as a treatment of spinal osteosarcoma is significantly constrained by the 
close proximity and the radiation tolerance of the spinal cord, that is about $45 \mathrm{~Gy}$, which is below the dose required to control most osteosarcomas with subclinical microscopic disease, microscopically positive margins and gross residual disease ${ }^{[1],[3] \text {, }}$ $[14],[16],[18]$. To reduce these toxicities produced due to the higher doses of radiation, intensity modulated radiation therapy (IMRT) emerged as a promising technique when surgery is not possible, because of its ability to spare normal tissues ${ }^{[13],[16]-[18]}$. It modulates the radiation dose and gives higher dose to the spinal tumour while limiting dose to the spinal cord ${ }^{[13],[16]-[18]}$.

Apart from IMRT there are other newer techniques such as, proton beams and heavy charged particles such as carbon ion radiation therapy. Proton beams can also be used as intensity modulation therapy which help in optimizing the dose distribution to the normal surrounding tissues and vital organs, offers advantages for the treatment of spinal $\mathrm{OS}^{[5],[16]}$. Carbon ion therapy having a large energy release on targets and lesser scattering in tissues achieves a good physical dose deposition and provides a satisfactory local control with inoperable cases of spinal $\mathrm{OS}^{[5],[16]}$. Therefore, situations in which resection is not technically possible or when the tumour has been resected with positive margins resulting in high risk of local failure, conventional and highly conformal RT techniques can result in achieving promising local control and palliation in patients with spinal $\mathrm{OS}^{[13]}$.

\section{Conclusions}

By using multimodal treatment approach, we achieved a favourable outcome in local control in a case of spinal OS, and have shown a significant relief in symptoms of compressive myelopathy after radiation therapy.

\section{Acknowledgement: NONE}

\section{References}

1. Schoenfeld AJ, Hornicek FJ, Pedlow FX, et al. Osteosarcoma of the spine: experience in 26 patients treated at the Massachusetts
General Hospital, The Spine Journal. 2010;10:708-714.

2. Durfee RA, Mohammed M, Luu HH et al. Review of Osteosarcoma and Current Management, Rheumatol Ther (2016) 3:221-243

3. Katonis P, Datsis G, Karantanas A et al. spinal osteosarcoma, Clinical Medicine Insights: Oncology 2013:7 199-208.

4. Ozaki T, Flege S, Liljenqvist U, et al. Osteosarcoma of the spine: experience of the Cooperative Osteosarcoma Study Group. Cancer. 2002;94(4):1069-77.

5. Kelley SP, Ashford RU, Rao AS, Dickson RA. Primary bone tumours of the spine: a 42-year survey from the Leeds Regional Bone Tumour Registry. Eur Spine J. 2007;16(3):405-9.

6. Chen DJ, Lai Q, Zhu J et al. lumbar spinal canal osteosarcoma a case report, Medicine. 2018;97:25(e11210).

7. Federman N, Bernthal N, Eilber FC et al. The multidisciplinary management of osteosarcoma. Current Treatment Options in Oncology. 2009;10:82-93.

8. Sundaresan N, Rosen G, Boriani $\mathrm{S}$ et al. Primary malignant tumors of the spine. Orthop Clin North Am 2009;40:21-36.

9. Klein MJ, Siegal GP et al. Osteosarcoma: anatomic and histologic variants. Am J Clin Pathol. 2006;125(4):555-81.

10. Fletcher CDM, Unni KK, Mertens F, World Health $\mathrm{O}$, International Academy of $\mathrm{P}$. Pathology and Genetics of Tumours of Soft Tissue and Bone: IARC Press; 2002.

11. Ilaslan H, Sundaram M, Unni KK, Shives TC et al. Primary vertebral osteosarcoma: imaging findings. Radiology. 2004; 230 (3):697-702.

12. Sciubba DM, Okuno SH, Dekutoski MB, Gokaslan ZL. Ewing and osteogenic sarcoma: evidence for multidisciplinary management. SPINE. 2009;34:S58-68.

13. DeLaney TF, Park L, Goldberg SI, et al. Radiotherapy for local control of 
osteosarcoma. Int J Radiat Oncol Biol Phys. 2005;61(2):492-8.

14. DeLaney TF, Liebsch NJ, Pedlow FX, et al. Phase II study of high-dose photon/proton radiotherapy in the management of spine sarcomas. Int J Radiat Oncol Biol Phys 2009; 74:732-9.

15. Hogendoorn PC, Athanasou N, Bielack S, De Alava E, et al. Bone sarcomas: ESMO Clinical Practice Guidelines for diagnosis, treatment and follow-up. Ann Oncol. 2010;21:204-213.

16. Patel S, DeLaney TF et al. Advancedtechnology radiation therapy for bone sarcomas. Cancer Control. 2008;15(1):2137.

17. Kaushal A, Citrin D et al. The role of radiation therapy in the management of sarcomas. Surg Clin North Am. 2008;88(3):629-46, viii.

18. DeLaney TF, Trofimov AV, Engelsman M, Suit HD. Advanced-technology radiation therapy in the management of bone and soft tissue sarcomas. Cancer Control. 2005;12(1):27-35. 